\title{
Acoustic properties of washing machines in terms of customer expectations
}

\author{
Anna Yehorova ${ }^{1, *}$, Ervin Lumnitzer ${ }^{1}$ \\ 1 Technical University of Kosice, Faculty of Mechanical Engineering, Department of Process and Environmental Engineering
}

\begin{abstract}
The presented paper focuses on the possibilities of evaluating the acoustic properties of partial noise sources of household washing machines. The introductory parts are focused on the analysis of the use of a new type of properties, so-called customer-oriented acoustic properties of the product. The next part focuses on the design of an evaluation methodology, which is used for laboratory measurements of psychoacoustic properties. This methodology, after laboratory testing, is used for measurements in real conditions of selling, directly in sales premises. A listening test is proposed, the results of which are used to determine the acoustic properties required by customers.
\end{abstract}

Keywords: Customer-oriented properties, sound, psychoacoustics, sound quality, listening test, laboratory measurements.

\section{Introduction}

Nowadays, the trend is for the products offered on the market to have a high technical level, for example, a long-term warranty is becoming commonplace. Today it is not a problem to produce a product with good technical parameters and the required service life, but to implement it on the market. For this reason, manufacturers need to focus on those features that have not been at the forefront of their interest so far, but have a certain added value and can increase customer interest in a particular product.

This process began with the advent of the so-called industrial product design, especially in the automotive industry. Later, preferences such as recyclability, energy optimization, adaptive and intelligent properties, acoustics, lighting properties, energy field optimization and many more began to be preferred. One of the attractive areas discussed in this paper is the acoustic design of products, which is related to a number of areas such as visualization of sound generation and propagation, psychoacoustics, simulation of sound propagation, sound perception and the influence of generated sound on human emotional state.

The issue of this paper is becoming increasingly important on a global scale. Several authors deal with the improvement of acoustic properties of various products in their works. Prezelj, J. in the article "Centrifugal fan with inclined blades for vacuum cleaner motor" [2] describes the possibilities of influencing the alternative geometry of the impeller of a centrifugal fan on its acoustic parameters. The author deals with the reduction of noise and the improvement of the product as a whole from the point of view of evaluation by using psychoacoustic parameters.

The process of optimizing the internal noise and vibration of vehicles with the help of sound quality parameters is characterized in the paper "A computational approach for optimizing vehicles' interior noise and vibration" by A.M. Al-Dhahebi [3]. 
Another author who dealt with psychoacoustics in his research was N. Valverde in the publication "Psychoacoustic metrics for assessing the quality of automotive HMls' impulsive sounds", where he characterizes the acoustic feedback of prototypes of mechanical buttons and parameterizes the sound when using them with acoustic and psychoacoustic sound parameters [4]. The relationship between consumer satisfaction and the psychoacoustic quality of electric vehicles is analysed in the paper "The relationship between consumer satisfaction and psychoacoustics of an electric vehicle signature sound" by D. J. Swart [5].

According to the standard STN EN ISO 9000: 2016 quality is "degree to which a set of inherent characteristics fulfils requirement". Quality can be defined as the ability to ensure that customer needs are met. It is clear that all these product quality requirements apply throughout the product life cycle.

In nowadays specific - customer-oriented product features have come to the fore. These properties cannot affect the safety and reliability of the product, they have no limits, but they significantly improve the "quality" properties required by customers, which increase the attractiveness of the product on the market and ultimately its marketability.

Customer evaluations may or may not be legally or normatively substantiated, for example, the aesthetic properties of a product are not specified anywhere. But now the customer has begun to prefer specific features of quality, which has caused the shift of these characteristics to the significant area.

The basic goal of the research carried out by the authors of the article is to propose a methodology for optimizing the acoustic parameters of products and to verify this methodology on a specific product. It is a development in the field of acoustic design and its implementation in the production process of a consumer product - an automatic washing machine.

The proposed methodology is goal-oriented for practical use in the process of optimizing the acoustic design of products, from the perspective of customer expectations.

\section{Methodology}

The proposed methodology is presented on the basis of the scheme of the product quality evaluation procedure. It shows the method of experiment and the sequence of steps leading to the evaluation of the product in terms of its acoustic quality.

After product selection, the following partial sources of washing machine sound were identified:

- opening and closing of the main door,

- opening and closing the detergent dispenser,

- tapping on the side wall of the washing machine.

Listed sound sources was not chosen randomly. They were selected on the basis of the results of surveys conducted abroad and with monitoring the behaviour of customers in choosing the monitored product.

Obviously, this behaviour was not always rational, often focusing on irrelevant, even irrational elements. A typical representative of such an element is the tapping on the side wall of the washing machine. It has no information value; it does not represent any technical parameter. It is debatable whether it represents the "overall" quality of the product. In Fig. 1 a scheme of the methodology for evaluating the acoustic quality of a product - automatic washing machine is shown.

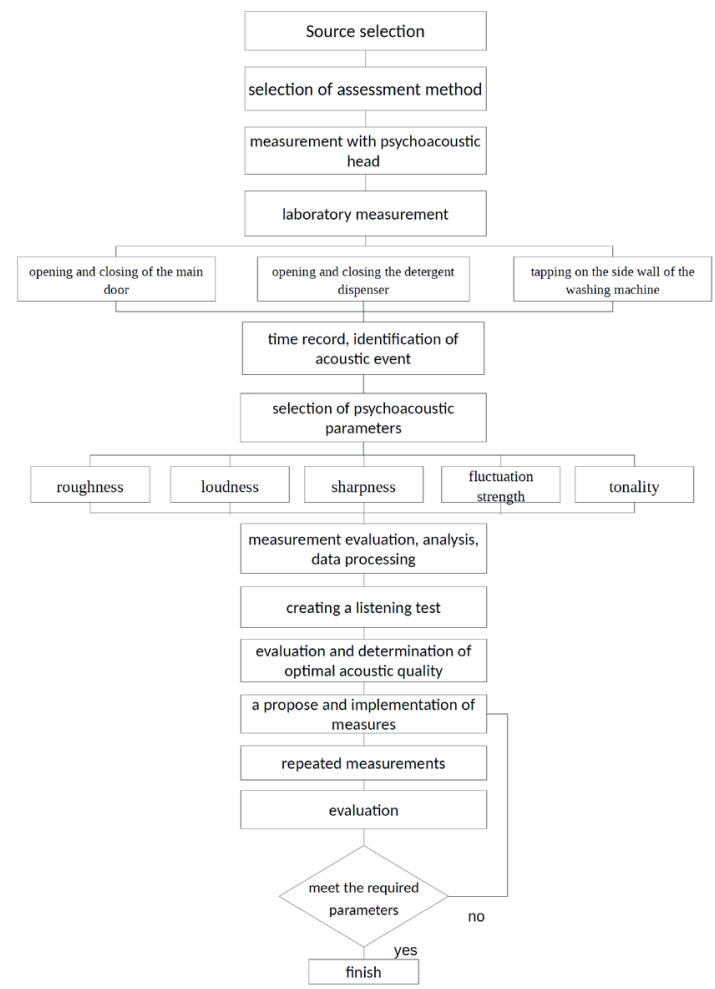

Figure 1: Scheme of methodology for evaluation of acoustic quality of automatic washing machine. 
To verify the proposed methodology, laboratory measurement of psychoacoustic parameters was performed using a binaural measuring system in a soundless chamber, designed to measure the acoustic parameters of household appliances in accordance with the relevant standard. Measurements were performed on one automatic washing machine.

Figure 2 shows the measuring point, the location of the sound source and the sensing part of the measuring device.

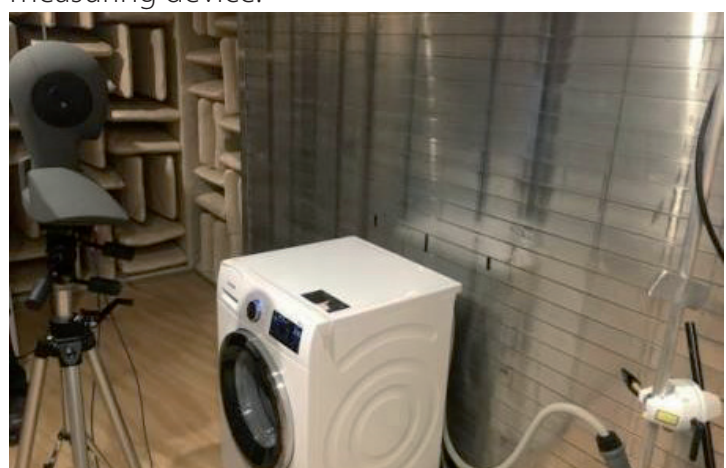

Figure 2: A scene of the measurement in the anechoic chamber.

\section{Verification of the proposed methodology in practice}

Based on the methodology given in the previous chapter, measurements were performed on 10 test samples, directly in the sales premises of automatic washing machines.

The measurement was carried out in the premises of a shopping centre focused on the sale of household appliances. For maximum objectivity, measurements were made before the store opened. Each washing machine was placed on a solid surface in a stationary position. The measurement situation is given in Fig. 3.

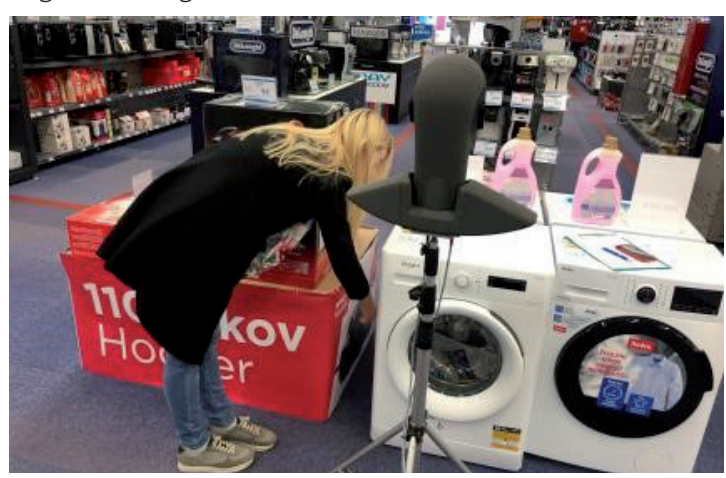

Figure 3: A view of a measuring point in a store.
Based on measurements performed in the anechoic chamber and in the sales premises, it was found that the process of opening the main door and the detergent dispenser was significantly less important in terms of psychoacoustic properties of the evaluated product than the process of closing the main door of the washing machine. For this reason, we only focused on the process of closing the main door during the evaluation.

Evaluations were performed for the left and right ear separately. For each monitored quantity, the average values of the examined acoustic event for the right and left ear are evaluated, as well as the maximum value of a specific psychoacoustic quantity. In addition to the above quantities, the frequency dependences of the observed sound expression obtained by FFT analysis were also evaluated. Since the two-channel sensing part of the measuring system was located in the axis of the measured washing machine, some values show small differences between the right and left channels. The differences are more significant especially if the examined sound source was outside the axis of symmetry of the product (detergent dispenser, knocking on the side wall of the washing machine).

Selected evaluation parameters were Loudness, Sharpness, Fluctuation Strength, Roughness and Tonality.

\subsection{Measurement results}

To clarify the results, only the results from the measurement and evaluation of one washing machine (washing machine no. 1) are given below. Summary results from the measurement and evaluation of all 10 washing machines are given in Tab. 1.

Figures 4 - 6 show the measured values of psychoacoustic parameters for the customeroriented features of the acoustic quality of the product - closing the main door, closing the detergent dispenser and tapping on the side wall of the washing machine.

Based on the above results from the measurement of washing machine no. 1 it can be stated:

- closing the main door - It is manifested by a high level of sound pressure, in this respect it is one of the worst construction solutions. High values are reflected in increased loudness, but sharpness and roughness show very small values. Significant tonal components are evident, which is reflected in high tonality; 
- closing the detergent dispenser - Loudness reaches one of the highest values compared to other examined washing machines. The values of other psychoacoustic quantities are high, except for the sharpness, which reaches an average value;

- tapping on the side wall of washing machine - Loudness reaches very high values, which are compensated by lower sharpness and roughness. The sound pressure level is the highest of all evaluated washing machines.

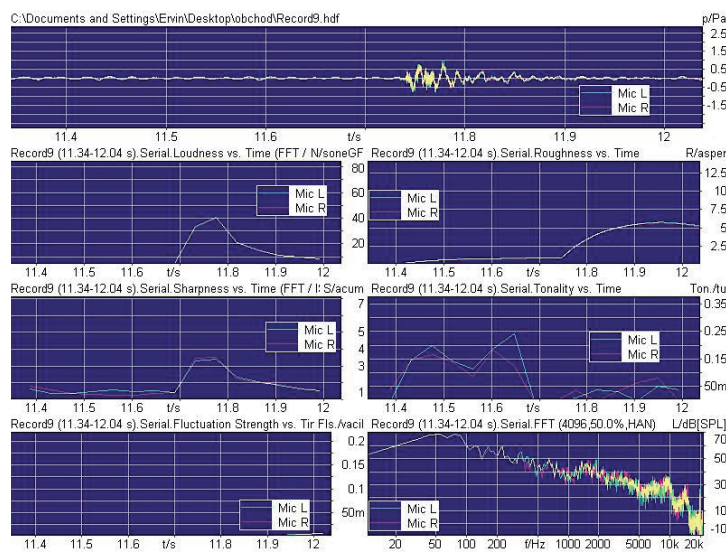

Figure 4: Psychoacoustic quantities when closing the main door of washing machine no.1.

Table 1 shows the summary results from the measurement of the monitored parameters of all 10 washing machines when closing the main door.

Table 1: Results of measuring psychoacoustic quantities when closing the main door of washing machines 1-10.

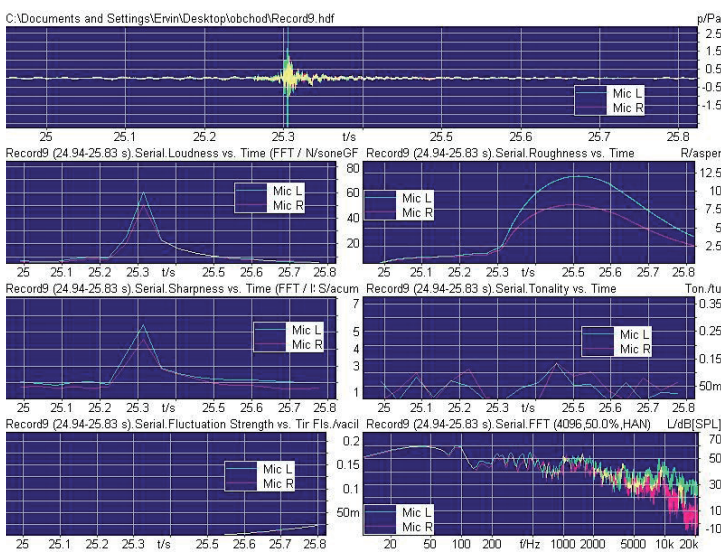

Figure 5: Psychoacoustic quantities when closing the detergent dispenser of washing machine no.1

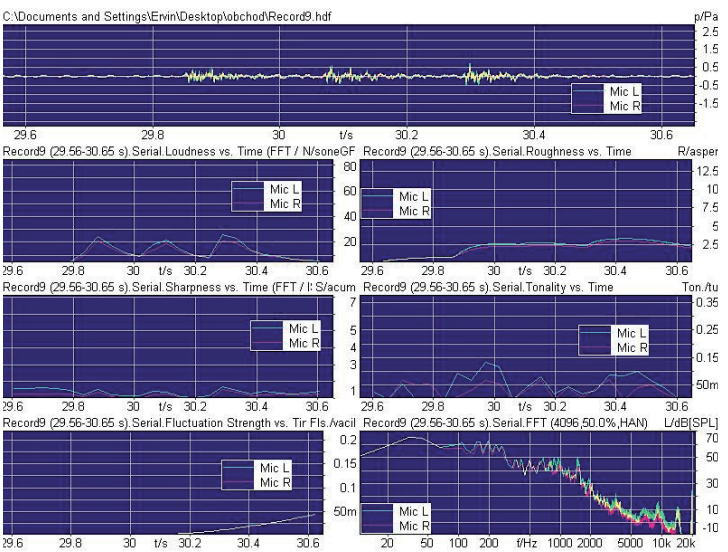

Figure 6: Psychoacoustic quantities when tapping on the side wall of washing machine no.1.

\begin{tabular}{|l|l|l|l|l|l|l|l|l|l|l|}
\hline $\begin{array}{l}\text { Psychoacoustic } \\
\text { quantity }\end{array}$ & \multicolumn{3}{l}{ Loudness [son] } & \multicolumn{3}{l|}{ Sharpness [acum] } \\
\hline Washing machine no. & Left ear & Right ear & Max & Left ear & Right ear & Max & Left ear & Right ear & Max \\
\hline 1 & 11,6 & 11,4 & 41,09 & 1,85 & 1,83 & 3,44 & 0,00105 & 0,00103 & 0,00745 \\
\hline 2 & 10,1 & 9,7 & 49,62 & 1,55 & 1,49 & 3,49 & 0,00120 & 0,01100 & 0,04779 \\
\hline 3 & 16,4 & 16,0 & 59,68 & 2,94 & 2,83 & 6,57 & 0,00795 & 0,00802 & 0,04137 \\
\hline 4 & 11,7 & 11,9 & 37,53 & 2,49 & 2,37 & 4,34 & 0,00022 & 0,00022 & 0,00061 \\
\hline 5 & 10,2 & 8,9 & 41,3 & 2,5 & 2,0 & 4,30 & 0,01980 & 0,01920 & 0,07002 \\
\hline 6 & 11,5 & 11,2 & 46,76 & 2,42 & 2,39 & 4,65 & 0,00089 & 0,00086 & 0,00210 \\
\hline 7 & 10,1 & 10,1 & 61,60 & 2,57 & 2,44 & 6,40 & 0,00490 & 0,00512 & 0,02548 \\
\hline 8 & 7,96 & 8,11 & 26,41 & 2,20 & 2,23 & 3,33 & 0,00140 & 0,00145 & 0,00864 \\
\hline 9 & 9,95 & 9,95 & 58,06 & 2,13 & 2,10 & 5,33 & 0,0137 & 0,0131 & 0,05615 \\
\hline 10 & 13,6 & 13,4 & 70,13 & 3,12 & 2,89 & 7,37 & 0,00771 & 0,00751 & 0,03889 \\
\hline
\end{tabular}




\section{The listening test}

An audio recording was created from the measured sound events obtained during the measurement of the monitored sound sources directly in the store. This recording was used in listening tests.

The aim of the experiment was to find out how the respondents react to the audio recordings of different types of washing machines in the specified conditions on the basis of "ONLY HEARING PERCEPTION". The test was attended by 20 respondents aged 20 - 35 years. During the test, respondents had to respond to various questions.

Question 1: Classify the heard recordings in the order where 1 - is the most pleasant and 6 - is the most unpleasant sound.

Question 2: The sound of which washing machine is the quietest? Question 3: Which washing machine would you buy?

The result of sound evaluation tests was a set of equations 1, 2 and 3, combining psychoacoustic quantities with the subjective perception of the sound of the "average" customer. At the same time, the limit values necessary to achieve the desired sound expression were empirically defined in the tests.

Pleasantness

$$
\begin{aligned}
& 21.0558-176.247 \times L-13.4578 \times S+ \\
& +79.1158 \times T \times R>0 \\
& \quad \text { Strength } \\
& -8.01+21.89 \log R+2.32 \times \log T>1 \\
& \quad \text { Sonance quality } \\
& 4.6598+0.23548 \times L-3.06548 \times R+ \\
& +0.02254 \times T \times S<2
\end{aligned}
$$

Legend: L - Loudness, S - Sharpness, T - Tonality, R Roughness.

Fluctuation Strength due to its complicated relationship with perception was not taken into account in these evaluations.

These equations are two-dimensional. However, for a real evaluation of the cross-tests of the components and easier interpretation of their results, it is more advantageous to define the "band" in which the psychoacoustic input parameters should be located.

\subsection{Results of the listening test}

- Opening and closing the main door of washing machine

Based on the results of the listening test, the worst and best washing machine was from the same manufacturer. The difference in sounds when closing the door results from the closing mechanism of the door, which had a different construction.
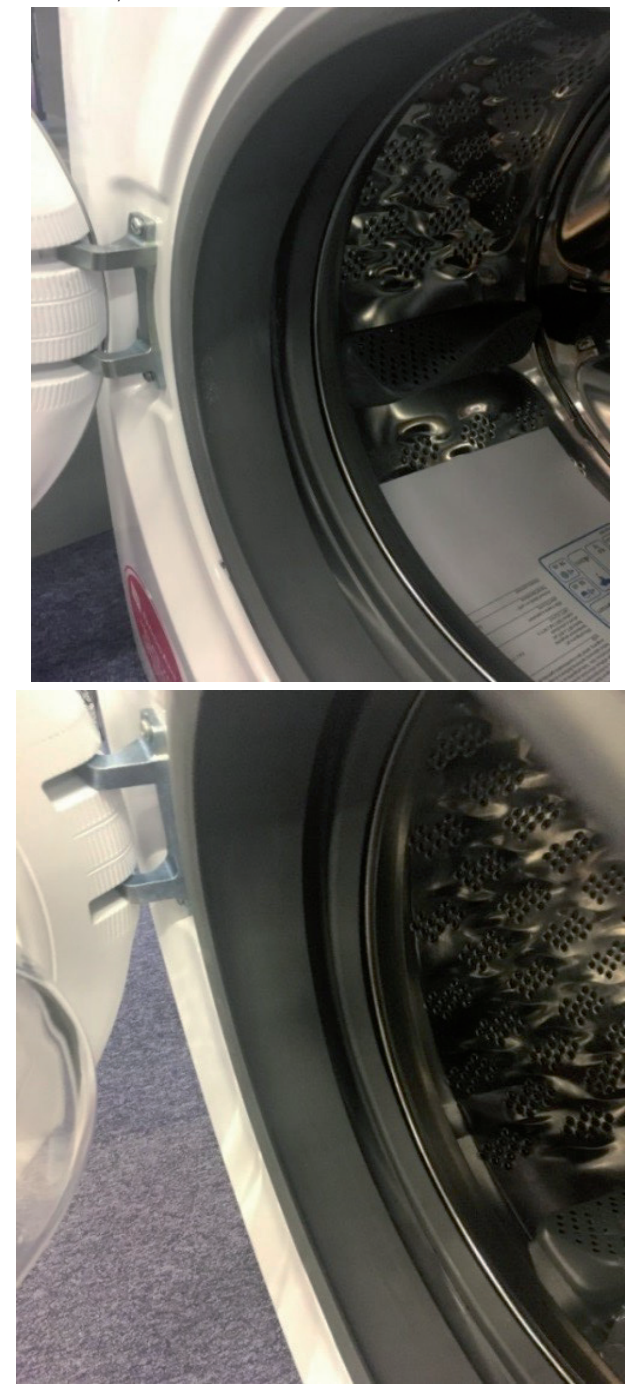

Figure 7: Comparison of the door closing mechanism.

- Opening and closing the detergent dispenser of washing machine

Based on the results of the listening test, washing machines that had retarders for closing the detergent dispenser were evaluated as the best.

\section{- Tapping on the side wall of washing machine}

The side walls of the monitored washing machines were made of sheets with various overhangs, which reduce the sound effects of the washing machine during its operation, especially during spinning. The results of the listening tests showed that the washing machine with the largest number of narrower overhangs was rated as the best. 

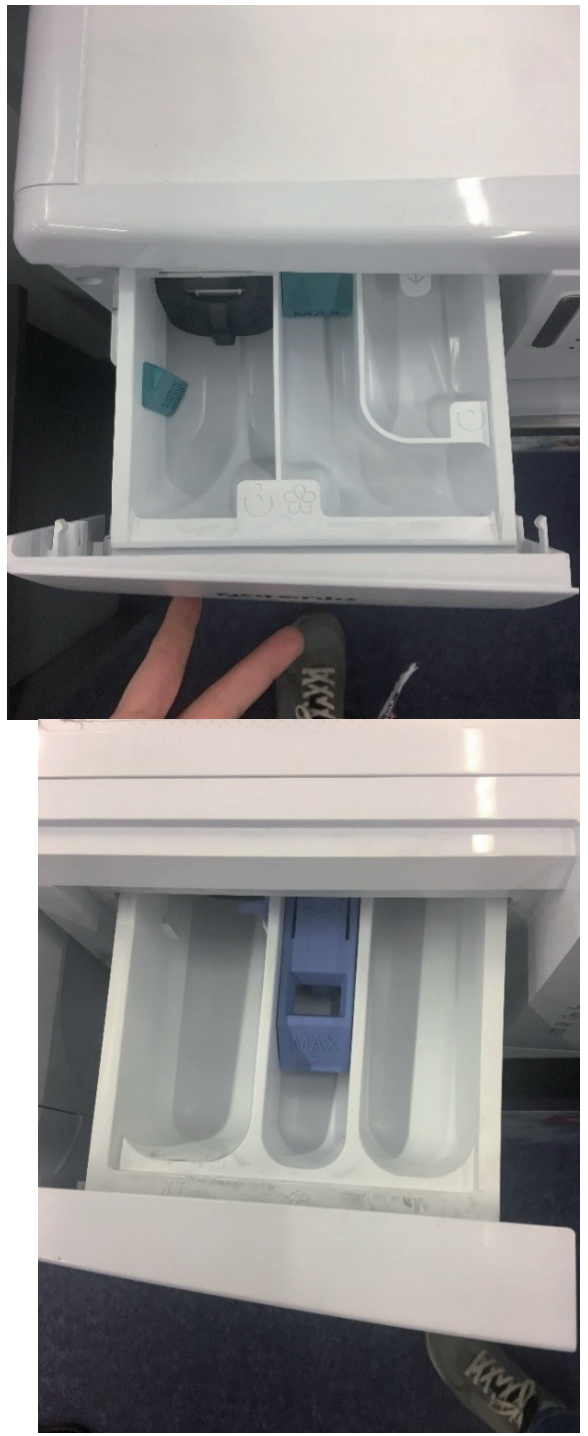

Figure 8: Comparison of the detergent dispenser.

\section{Conclusions}

In nowadays a lot of manufacturers strive to ensure that their products reach a high technical level on the one hand and that they also meet various specific customer requirements on the other. For this reason, manufacturers are beginning to focus on features that have not been at the forefront of their interest so far. The performed measurements and their evaluation confirm that these parameters, although they have no effect on the functionality of the product, differ significantly from each other in the subjective evaluation by potential customers. The performed experiment is used to determine recommendations for the

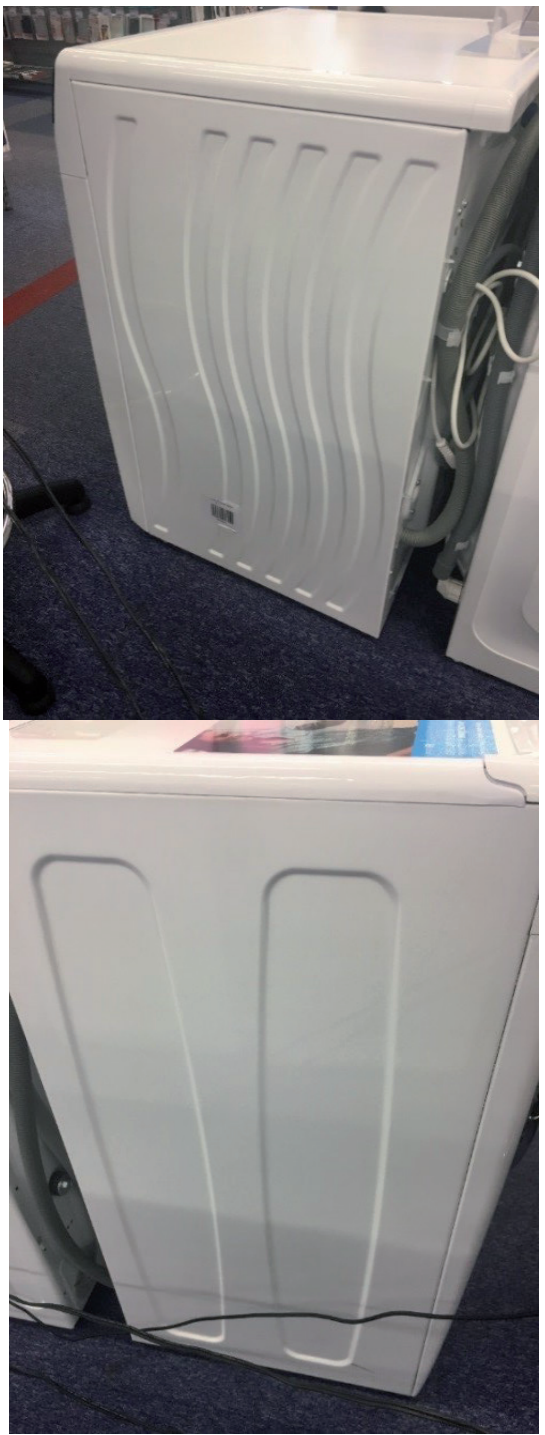

Figure 9: Comparison of washing machine side walls.

manufacturer of washing machines who want to improve these customer-oriented features and thus increase the marketability of their product.

\section{Acknowledgments}

\section{This paper was created within the project KEGA 032 TUKE-4/2018.}

\section{References and Notes}

[1] Lumnitzer, E. et al. (2012). Acoustics. Basics of psychoacoustics (in Slovak language). Elfa, s.r.o., Kosice. ISBN 978-80-8086-182-8

[2] Prezelj, J; Novakovic, T. (2018). Centrifugal fan with inclined blades for vacuum cleaner motor. Applied acoustics, vol: 140, pp. 13-23 
[3] Al-dhahebi, A.M; Junoh A.K; Mohamed Z. et al. (2017). A computational approach for optimizing vehicles' interior noise and vibration. International journal of automotive and mechanical engineering, vol. 14, issue: 4, pp: 4690-4703

[4] Valverde, N. et al. (2018). Psychoacoustic metrics for assessing the quality of automotive HMls'impulsive sounds. Applied Acoustics, vol. 137, pp. 108-120.

[5] Swart, D.J; Bekker, A. (2019). The relationship between consumer satisfaction and psychoacoustics ofelectric vehicle signature sound. Applied Acoustics, vol. 145, pp. 167-175.

[6] Lumnitzer, E. et al. (2014). Evaluation of the effects of physical factors on human health (in Slovak language). TU, Košice. ISBN 978-80-553-1632-1

[7] Lumnitzer, E; Ondrejčák, J. (2014). Sound analysis on electric kettle. Annals of Faculty Engineering Hunedoara International Journal of Engineering, vol. 12, no. 4 pp. 19-22.

[8] Lumnitzer, E; Liptai, P; Dolník, B; Benčová, B; Yehorova, A. (2019). The analysis of the impact of communicators on the health of employees. Acta Technica Corviniensis : Bulletin of Engineering, vol. 12, no. 2 pp. 45-48.

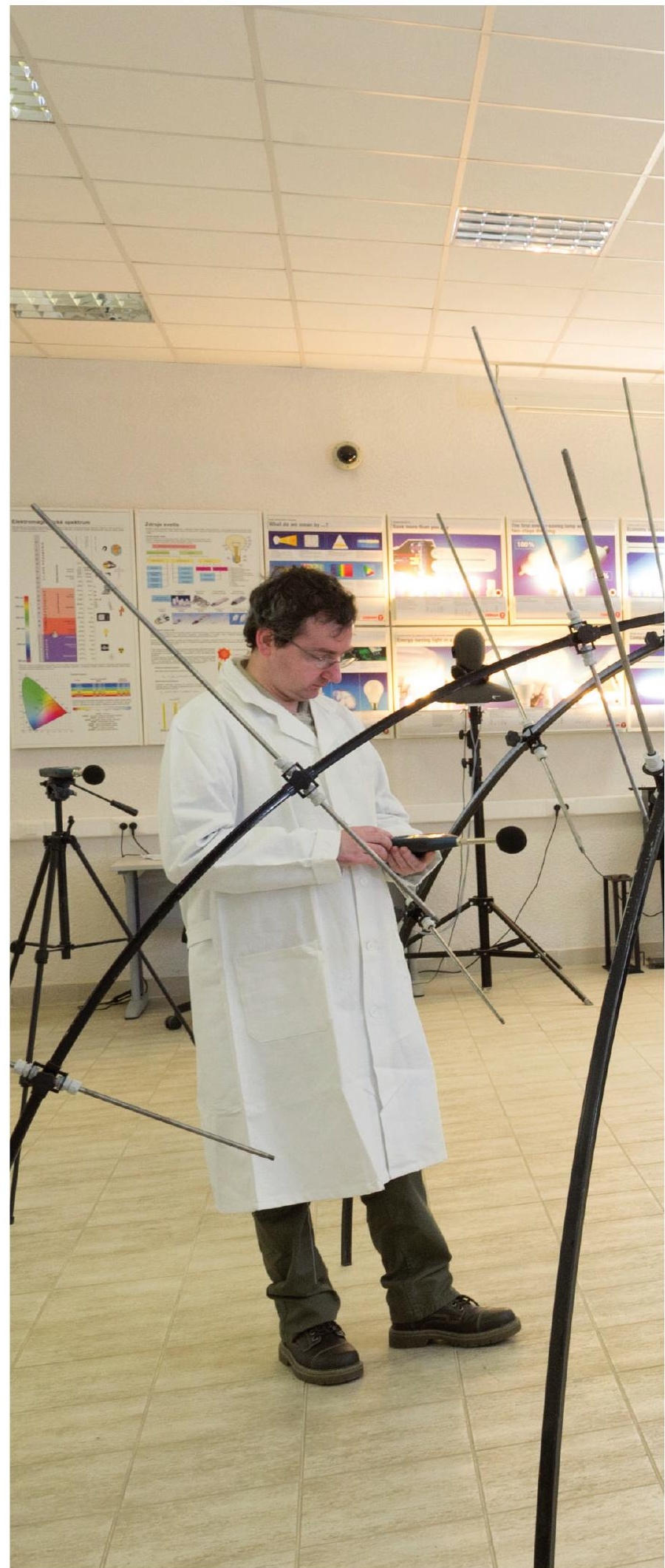

